\title{
DeVElopment of a RESpiration RATE METER -A Low-COST Design APPROACH
}

\author{
Souvik Das \\ Department of Biomedical Engineering \\ JIS College of Engineering, West Bengal, India \\ souvik. journals@gmail.com
}

\begin{abstract}
Measurement of physiological parameters like respiration rate is crucial in field of medicine. Respiration rate can indicate the state of rhythmic behaviour of heart and proper gaseous exchange in blood. As per Medical research, respiratory rate is regarded as the marker of pulmonary dysfunction. Respiration rate meters are used in measuring $\mathrm{CO}_{2}$ in expired air and in apnea detectors. It is also used in daily physiological tests like stress-o-meter for assessing ones level of stress that he/she can perceive in life after monitoring respiration rate, pulse rate and heart rate. This paper shed lights on the development of a lowcost respiration rate meter using infrared sensing and associated digital electronic circuitry. The proposed device is able to measure respiration rate in the range of 0-999 respirations/minute.
\end{abstract}

\section{KEYWORDS}

Respiration Rate Meter, Biomedical Device, Physiological Stress, Medical Electronics, Apnea Detector, Digital Circuits

\section{INTRODUCTION}

The respiration rate is a vital physiological parameter that helps to provide significant information about the health status of a patient, particularly that of the human respiratory system. Abnormal respiratory rate could indicate a variety of conditions including respiratory diseases as well as systemic abnormalities including cardiovascular abnormalities and acidosis. The respiratory rate is also a commonly used parameter in routine patient monitoring to detect early disease and deterioration in clinical conditions. This measurement is even important in vulnerable patients, e.g. critically ill, neonates, infants and the elderly [1]. For example, respiratory rate is a useful indicator in severe asthma $[2,3]$. The dynamic monitoring of human respiratory status during sleep imparts an important role in the diagnosis and treatment of sleep apnea, various sleep disorders and sudden death syndrome $[4,5]$. Despite its importance and widespread utility, there is a lack of simple respiratory rate measurement instruments that can be applied in clinical practice. In most centers today, manual measurement of respiratory rate is still routinely practiced and this is time consuming and labor intensive. The major function of the human respiratory system is to supply an adequate amount of oxygen to the human body to produce energy and maintain a proper acid-base balance by removing carbon dioxide from it. The respiration rate monitoring is very much significant in medical monitoring system as it plays an important role in assessing a variety of illnesses [6, 7].

The theory and practice of closed breathing systems evolved with the discoveries of oxygen and carbon dioxide and formed an integral part of the classic work of Lavoisier published between

DOI: $10.5121 /$ hiij.2013.2202 
1774 and 1785. This identified respiration as the uptake of oxygen and the excretion of carbon dioxide and formed the basis of general physiology [8]. With the advent of medical science and technology, several techniques have been adopted and also being proposed based on research works regarding the measurement of various physiological parameters including respiration rate. In the measurement of low-frequency biomedical parameters, such as heart rate and respiration rate, it is often necessary to measure the instantaneous frequency of their occurrence. Any rate measuring system based on the measurement of the time interval must, therefore, include a circuit to perform the necessary inverting function $[9,10]$. Khandpur et al. described a circuit for beatto-beat heart rate measurement and the circuit can be modified to measure respiration rate as well [9]. Robert and Thomas described a model to predict respiration from VCG [11]. The gold standard for respiratory rate estimation is through the measurement of respiratory flow using a pneumotach or using a capnograph [2, 12]. Iamratanakull et al. studied the estimation of respiration from physiologic pressure signals [13]. Brady et al. focused on garment-based monitoring of respiration rate using a foam pressure sensor [14]. A system for wearable respiration monitoring system based on digital respiratory inductive plethysmography has been described by We et al. [4]. In their design of respiration rate meter, Vasu et al. and Choi et al. used radio frequency sensors [15, 16], whereas Miwa and Sakai [17] proposed the use of body sound for the development of a heart rate and respiration measurement system. Implementation of optical methods, ultrasonic proximity sensor, impedance plethysmography, ECG signal (via wavelet), facial tracking method and real-time vision based system are also studied by Scalise et al. [18], Min et al. [19], Ansari et al. [20], Santo and Carbajal [21], Khalidi et al. [6] and Tan et al. [1] respectively regarding the measurement of respiration rate. Recent studies on respiration rate monitoring using capacitive textile force sensors, stepped-FM method, empirical mode decomposition method and laser Doppler vibrometry have also been carried out by Hoffmann et al. [22], Otsu et al. [23], Madhav et al. [24] and Scalise et al. [25].

However, despite the proposal and implementation different respiration rate monitoring system and ongoing studies regarding the same, there still exist some physiological monitoring instruments, which are not only expensive but also inconvenient and are not practical for extended monitoring. Therefore a more practical and simple method is needed for continuous respiratory rate monitoring. With this concern, an attempt is made to design a low-cost respiration rate meter using infrared sensor and simple digital electronics circuit components.

\section{Methodology}

\subsection{System Configuration}

The block diagram of the designed digital respiration rate meter is shown in Fig. 1. This meter uses a displacement transducer in combination with infrared (IR) transmitter and IR -receiver, respectively, for sensing the respiration rate. In the capillary glass tube of the physical interface (Fig. 5) of the meter, a light ball moves up and down during respiration process caused by Inhaling and exhaling the air. The IR-transmitter and receiver circuit assembly sense the ball movements and convert them into pulses by the pulse generator. A counter counts these pulses for a minute. A 3-digit LED (Light emitting diode) display monitors the respiration rate through a 7segment driver/decoder. To reset the display to initial state (zero) and activate the counter for 1minute to count the respiration pulse, switch S1 (the start switch) is used. The gate pulse generator utilises a monostable multivibrator that generates gating pulse of 1-minute duration, when triggered by start switch. 


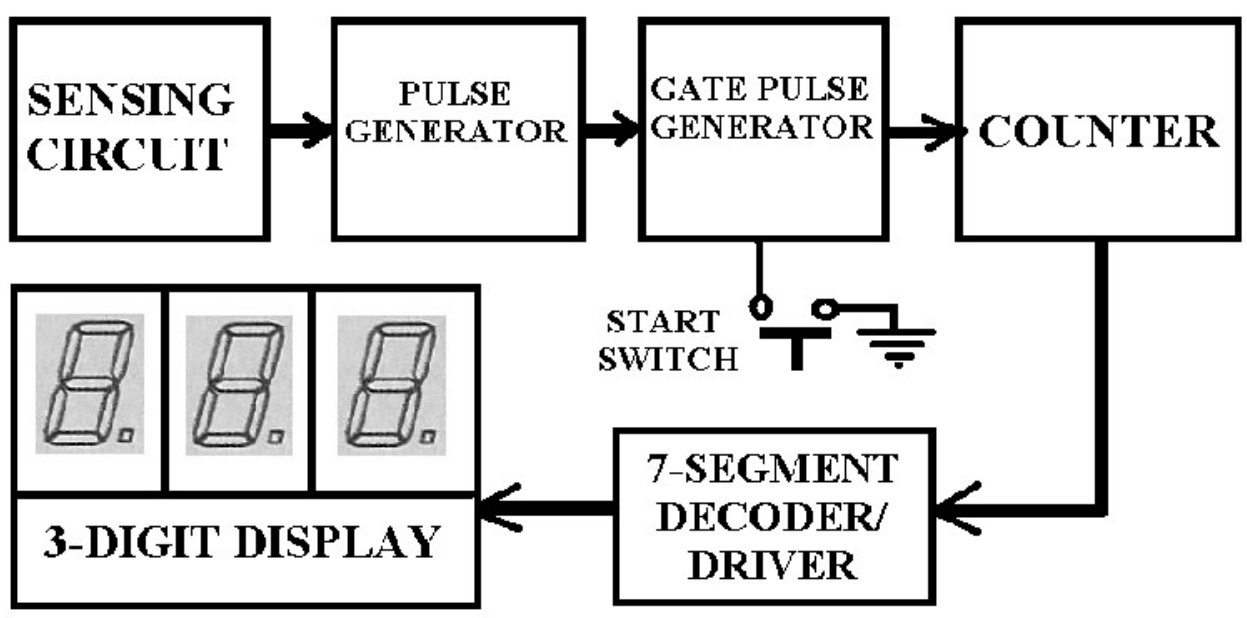

Fig. 1: Block diagram of digital respiration meter

\subsection{Circuit Configuration}

The circuit diagram for the digital respiration meter, designed is shown in Fig.2

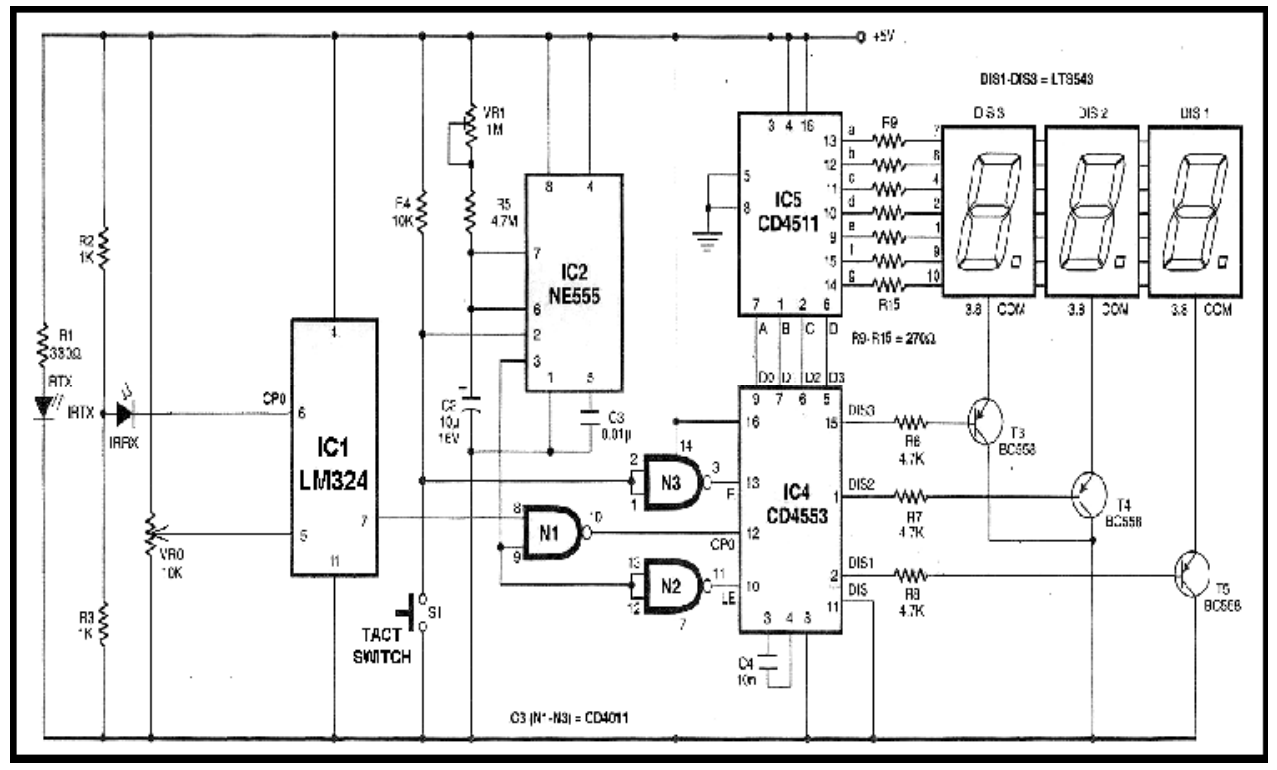

Fig. 2: Circuit diagram of digital respiration meter

In the above figure (Fig. 2), the IR-LED transmitter (designated as IRTX) joined in series with resistor R1 transmits infrared signal. The transmitted signal is received by the IR-LED receiver (designated as IRRX). The IR receiver is connected to pin6 of operational amplifier LM324 via a voltage divider network made through Resistor constructed by the resistors R2 and R3. As the transmitted IR signal is directly incident on the forward biased IR diode, it generates an electrical signal according to the IR intensity. Here, LM324 acts as a comparator and calibrated using the potentiometer (VR0) in such a way that it would output a single pulse whenever the transmission path between the IR transmitter and the receiver is blocked. It implies that a pulse is produced 
during inhalation and exhalation, whenever the ball crosses the IR beam. Two pulses are produced as the ball crosses the IR beam twice: one during inhalation and the other during exhalation. The output of LM324 acts as the clock pulse for 3-digit BCD counter CD4553 (IC4). The input pin 8 of the NAND gate N1 of IC3 is connected to the output of counter IC1. The output of monostable multivibrator (NE555) is shorted with the input pin 9 of NAND gate N1. The values of the resistor R5 and the preset VR1 are taken in such a way that the monostable multivibrator generates a pulse of 1-minute time duration.

Whenever the switch $\mathrm{S} 1$ is pressed, it triggers the monostable multivibrator and produce the pulse of 1-minute time period. This pulse is the input to the pin 9 of the NAND gate (N1). Thus, the NAND gate becomes open for 1-minute and the clock pulses from LM324 are passed to pin 12 of the 3-digit BCD counter (CD4553). The NAND gate, N2 controls the latch enable (LE) of the BCD counter and inverts the 1-minute pulse from NE555. The counter begins its counting function, as the LE of the counter goes low resulting from the high output of NE555. Again, the counter stops counting when the LE of the counter becomes high, caused by the low output from the timer IC (NE555). At this stage, the 7-segment LED display would show no change in the count. Initially, a '000' is shown on the 7-segment display, when the S1 is pressed. The IC CD4533 incorporates 3-negative edge triggered synchronously cascaded BCD counters. Each of these counters contains a quad latch at its output and each quad latch allows the storage of digit counting. Time-division-multiplexing (TDM) scheme is applied to outgoing information from the $\mathrm{BCD}$ counter so that only one BCD digit/number is displayed at a time. Digit-Select outputs provide display control. All the outputs are TTL (i.e. Transistor-Transistor-Logic) -compatible. The output selector of multiplexer is driven by the low frequency scanning clock pulse that is provided by an on-chip oscillator. The 7-segment decoder/driver IC, CD4511 receives the BCD count outputs from the counter IC (CD4533) and displays the count on the 3-digit 7-segment displays (DIS1 through DIS3). Resistors R9 through R15 are used for limiting the current.

The power supply circuit for the circuit shown in figure 1 is based on the circuit shown in Fig. 3



Fig 3: Power supply circuit for the digital respiration meter

The $230 \mathrm{~V}$ AC is stepped down by a transformer to provide the secondary output of $9 \mathrm{~V}, 500 \mathrm{~mA}$. A full-wave bridge rectifier having diodes D1 through D4 rectifies the output of the transformer. The rectified output is then filtered (by capacitor, C5) and regulated (by IC 7805, IC6), respectively. Ripples those might be present in the regulated power supply are bypassed by the 
capacitors C6 and C7. LED1 indicates the power 'ON' and the current through LED1 is limited by the resistor R17. The diode D5 would protect the regulator IC if, anyhow, its input becomes shorted to ground. Till now, the circuit has been developed on a breadboard as shown in Fig. 4. The circuit would be designed on a Vero board along with the physical interface assembly as shown in Fig. 5.

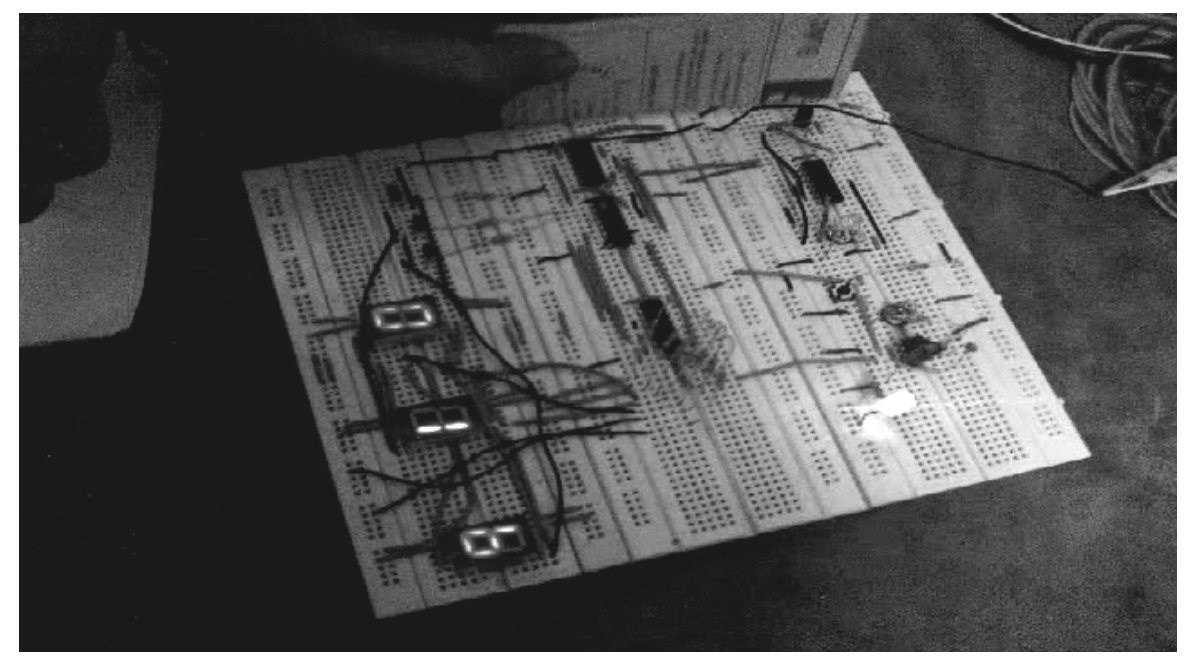

Fig. 4: Testing of the designed circuit on the bread board

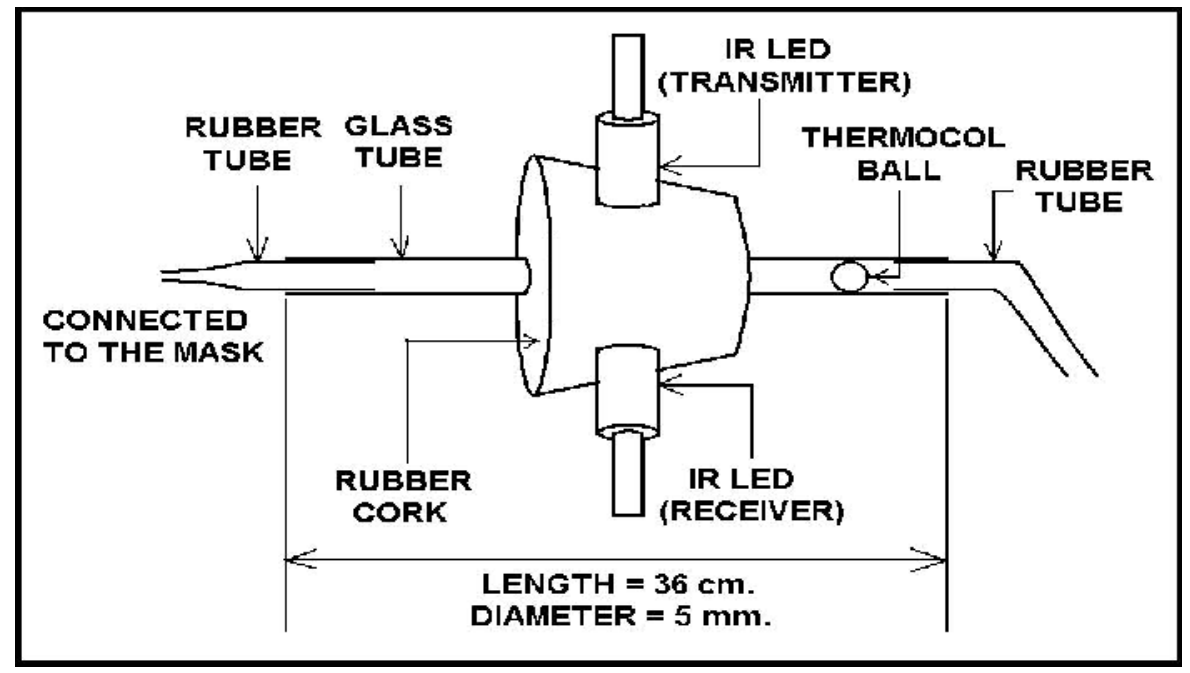

Fig. 5: The physical interface assembly

The physical interface assembly utilises a nose mask that is easily available in medical shops. This is mainly used in hospitals for supplying oxygen to the patients for respiration.

\section{RESULTS \& DiSCUSSIONS}

Since the designed circuit is at its developmental stage and since the physical interface assembly is going to be implemented over it, subject data have not been yet collected. However, the fact that the designed circuit has run satisfactorily was proved when it was tested on the breadboard 
Health Informatics - An International Journal (HIIJ) Vol.2, No.2, May 2013

which would be replaced by Vero board. The following series of figures in figure 6 (6A to 6B) obtained during the testing the circuit on the breadboard proved its ability to count pulses. There are only 8 (starting from 000 to 007) stages have been shown here, although pulses counting were tested for 1 minute.



$6 \mathrm{~A}$



$6 \mathrm{C}$

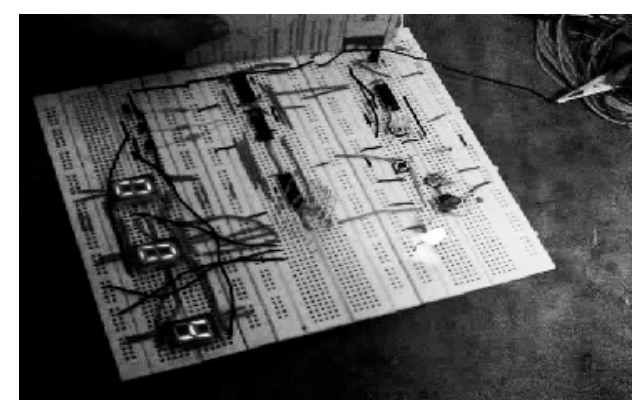

$6 \mathrm{E}$

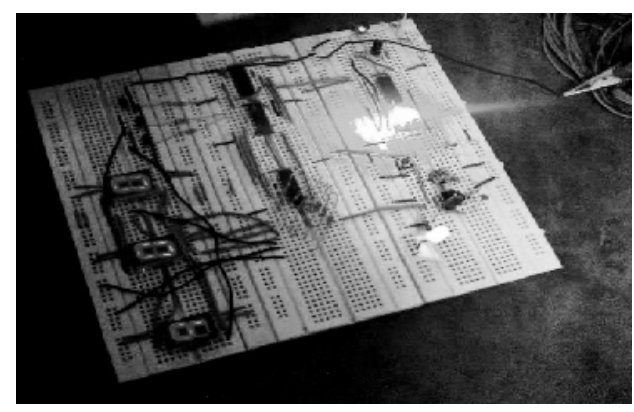

$6 \mathrm{G}$

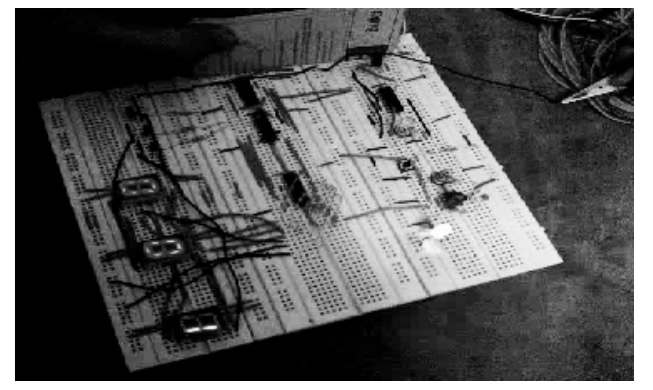

$6 \mathrm{~B}$

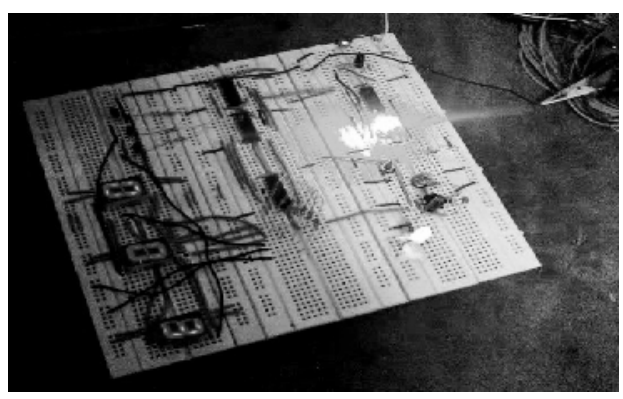

$6 \mathrm{D}$

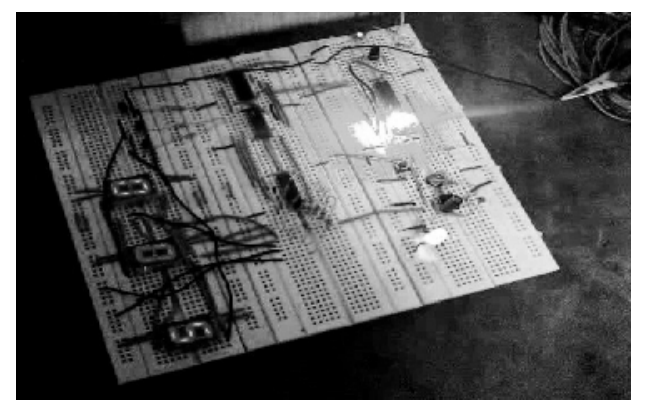

$6 \mathrm{~F}$

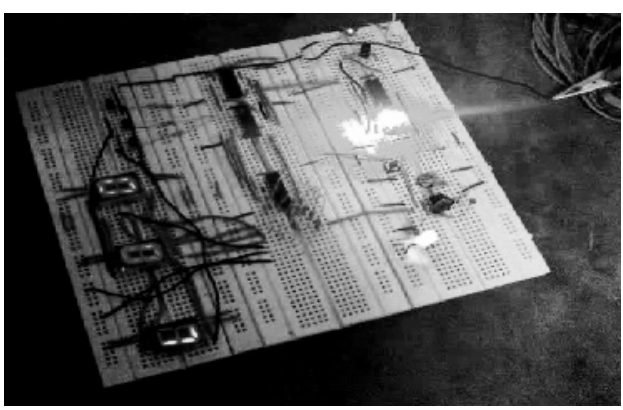

$6 \mathrm{H}$

Fig. 6A-6H: Testing the respiration rate measurement circuit on Vero board 


\section{Conclusions}

It is to be mentioned here that digital respiration rate meter circuit for designed on the breadboard proved its functioning as desired. The circuit is going to be implemented on veroboard along with the physical interface to make it operative for practical use in the healthcare field. The major advantage of this monitoring device is its low-cost for which it will get an easy access to monitor respiration rate (one of the significant physiological parameters) to not only in medical field but also at home. The reason behind is the handling the device for which one need not to be strictly from medical background. The above mentioned device can also be modified with additional hardware circuitry so that it would not only monitor human respiration rate but also other significant physiological parameters (e.g. blood pressure, temperature etc.).

\section{ACKNOWLEDGEMENTS}

I would like to convey my special and sincere thanks and gratitude to Dr. Asit Guha, Director, JIS College of Engineering, Kalyani, Nadia (West Bengal) for providing me the opportunity and platform to design the system.

\section{REFERENCES}

[1] K.S. Tan, R. Saatchi, H. Elphick, and D. Burke: Real-Time Vision Based Respiration Monitoring System, 7th International Symposium on Communication Systems Networks and Digital Signal Processing (2010), p. 770-774.

[2] J. Zhang, and D.Y.T. Goh: A Novel Respiratory Rate Estimation Method for Sound-Based Wearable Monitoring Systems, 33rd Annual International Conference of the IEEE EMBS (2011), p. 32133216.

[3] S Kesten, R Maleki-Yazdi, BR Sanders, JA Wells, SL McKillop, KR Chapman and AS Rebuck: Respiratory rate during acute asthma, Chest, Vol. 97(1) (1990), p. 58-62.

[4] D. Wu, L. Wang, , Y.T. Zhang, B.Y. Huang, B. Wang, S.J. Lin, and X.W. Xu: A Wearable Respiration Monitoring System Based on Digital Respiratory Inductive Plethysmography, 31st Annual International Conference of the IEEE EMBS (2009), p. 4844-4847.

[5] X. Zhu et al.: Real-time Monitoring of Respiration Rhythm and Pulse Rate During Sleep, IEEE Trans. Biomed. Eng., Vol. 53 (2006).

[6] FQ AL-Khalidi, R. Saatchi, D. Burke and H. Elphick: Facial Tracking Method for Noncontact Respiration Rate Monitoring, 7th International Symposium on Communication Systems Networks and Digital Signal Processing (2010), p. 751-754.

[7] B. Mazzanti et al.: Validation of an ECG-derived respiration monitoring method, Computers in Cardiology, Vol.30 (2003), pp.613-616.

[8] D.C. White: The History and Development of Low Flow Breathing Systems. IEE Seminar, Low Flow Anaesthesia Breathing Systems - Technology, Safety and Economics (1999), p.1.

[9] S.C. Nijhawan, V.K. Sharma and R.S. Khandpur: A Beat-to-Beat Heart Rate Meter, IEEE transactions on biomedical engineering, Vol. BME-28 (1981), p. 42-44.

[10] M.L. Fichtenbaum: Counter inverts period to measure low frequency, Electronics, Vol. 49 (1976), pp. 100.

[11] R.C. Wang and T.W. Calvert: A Model to Predict Respiration from VCG Measurements, Proceedings of IEEE Conference on Decision and Control and 11th Symposium on Adaptive Processes (1972), p. 14-18.

[12] R. Ellis et al.: Comparative review of techniques for recording respiratory events at rest and during deglutition, Dysphagia, Vol. 12 (1997), p. 24-38.

[13] S. Iamratanakull, J. McNamesl and B. Goldstein: Estimation of Respiration from Physiologic Pressure Signals, Proceedings of the 25th Annual International Conference of the IEEE EMBS (2003), p. 2734-2737. 
Health Informatics - An International Journal (HIIJ) Vol.2, No.2, May 2013

[14] S. Brady, L.E. Dunne, R. Tynan, D. Diamond, B. Smyth, G.M.P. O’Hare: Garment-Based Monitoring of Respiration Rate Using a Foam Pressure Sensor, Proceedings, Ninth IEEE International Symposium on Wearable Computers (2005), p. 214-215.

[15] V. Vasu, N. Fox, T. Brabetz, M. Wren, C. Heneghan and S. Sezer: Detection of Cardiac Activity using a $5.8 \mathrm{GHz}$ Radio Frequency Sensor, 31st Annual International Conference of the IEEE EMBS (2009), p. 4682-4686.

[16] J.H. Choi, and D.K. Kim: A Remote Compact Sensor for the Real-Time Monitoring of Human Heartbeat and Respiration Rate, IEEE transactions on biomedical circuits and systems, Vol. 3 (2009), p. 181-187.

[17] H. Miwa and K. Sakai: Development of heart rate and respiration rate measurement system using body-sound, Proceedings of the 9th International Conference on Information Technology and Applications in Biomedicine (2009), p. 1-4.

[18] L Scalise, P Marchionni and I Ercoli: Optical Method for Measurement of Respiration Rate, Proceedings, IEEE International Workshop on Medical Measurements and Applications (2010), p. 1922.

[19] S.D. Min, J.K. Kim, H.S. Shin, Y.H. Yun, C.K. Lee, and M. Lee: Noncontact Respiration Rate Measurement System Using an Ultrasonic Proximity Sensor, IEEE sensors journal, Vol. 10 (2010), p. 1732-1739.

[20] S. Ansari, A. Belle, K. Najarian and K. Ward: Impedance Plethysmography on the Arms: Respiration Monitoring, IEEE International Conference on Bioinformatics and Biomedicine Workshops (2010), p. 471-72.

[21] A.E. Santo and C. Carbajal: Respiration Rate Extraction from ECG Signal via Discrete Wavelet Transform, Circuits and Systems for Medical and Environmental Applications Workshop (2010), p. $1-4$.

[22] T. Hoffmann, B. Eilebrecht, and S. Leonhardt: Respiratory Monitoring System on the Basis of Capacitive Textile Force Sensors, IEEE sensors journal, Vol. 11(2011), p. 1112-1119.

[23] M. Otsu, R. Nakamura, and A. Kajiwara: Remote Respiration Monitoring Sensor Using Stepped-FM, IEEE Sensors Applications Symposium (2011), p. 155-158.

[24] K.V. Madhav, M.R. Ram, E.H. Krishna, N.R. Komalla, K.A. Reddy: Estimation of Respiration Rate from ECG, BP and PPG signals using Empirical Mode Decomposition, IEEE Instrumentation and Measurement Technology Conference (2011), pp. 1-4.

[25] L. Scalise, I. Ercoli, P. Marchionni and E.P. Tomasini: Measurement of Respiration Rate in Preterm Infants by Laser Doppler Vibrometry, IEEE International Workshop on Medical Measurements and Applications Proceedings (2011), p. 657-661.

\section{AUTHOR}

Mr. Souvik Das did his M.E. in Biomedical Engineering from Jadavpur University, West Bengal and B.Tech in Electronics \& Instrumentation Engineering from University of Kalyani, West Bengal. His research interests include medical device development, sensor development, patient care monitoring and biomechanics. He has 6 years of teaching and 1 year research experience. He has published 10 research papers in National and International Journals, conferences and seminars. He has also participated and presented research papers in various National and International Conferences/ seminars/ symposiums etc. He is member of IAENG, IACSIT, ISDWT


and ISCOC. 